\title{
Abdominal and pelvic surgeries in patients with irritable bowel syndrome - the possible relevance of their associated psychiatric and psychological symptoms
}

\author{
Alin Ciobîcă, Peiu Sorin Nicolae, Daniel Timofte
}

\begin{abstract}
Alin Ciobîcă - PhD, Department of Research, Faculty of Biology, Alexandru Ioan Cuza University, Iasi, Romania, Academy of Romanian Scientists, Bucuresti, Romania, Center of Biomedical Research, Romanian Academy, Iaşi, Romania

Peiu Sorin Nicolae - MD, Faculty of Medicine, "Grigore T. Popa" University of Medicine and Pharmacy, Iaşi, Romania

Daniel Timofte - MD, PhD, professor, "Grigore T. Popa" University of Medicine and Pharmacy, Iaşi, Romania
\end{abstract}

\begin{abstract}
Irritable bowel syndrome (IBS) is characterized by a chronic functional disorder of the gastrointestinal system. Despite the efforts, to this day no biomarker has been found, so IBS is still only diagnosed clinically. Patients suffering from IBS receive disproportionately high rates of pelvic and abdominal surgeries. In the present article we reviewed the available literature regarding three of the most common surgical procedures (appendectomy, cholecystectomy and hysterectomy) which are associated with IBS symptoms. The mechanisms through which IBS may predispose to these disorders are still to be elucidated. However, it has been speculate that patients suffering from IBS often present hypersensitivity for visceral sensation. This hypersensitivity may explain why IBS is leading to possible misdiagnosis of appendicitis. In addition, constipation and a specific subtype of IBS- pain were more common in individuals who underwent hysterectomy. An explanation on the observed high rate of cholecystectomy in IBS population is related to the gastrointestinal disturbances and abdominal pain that is fairly common after cholecystectomy. We recommend that the history of IBS is taken into consideration before performing any pelvic or abdominal surgery. CT scans should also be performed in these groups of patients especially if these patients also suffer from IBS. Also the psychiatric and psychological relevance of their symptoms (which are quite commune in IBS, as our group previously described) is discussed in the present context.
\end{abstract}

KEYWORDS:

Irritable bowel syndrome, IBS, surgery, appendectomy, cholecystectomy, hysterectomy, psychiatric and psychological symptoms. 


\section{INTRODUCTION}

Irritable bowel syndrome (IBS) is characterized by a chronic functional disorder of the gastrointestinal system. Individuals who suffer from this disorder describe their symptoms as abdominal pain and altered bowel habit, with either predominantly diarrhea (IBS-D), constipation (IBS-C), or a combination of the two (IBS-M). Interestingly, despite the efforts to this day no biomarker has been found, so IBS is still only diagnosed clinically.

Regarding the history of this disorder, the first reports in which IBS is described dates back to the very beginning of the 20th century (1). In the early beginnings, diagnosing IBS was only made by excluding any other possible malignant, inflammatory or infectious disease (2). Furthermore, this illness continued to be frequently misdiagnosed and poorly understood well into the 1970s, adding the problem of unnecessary and ineffective surgeries which were performed for a remedy IBS.

The first effort to standardize IBS symptoms in order to reduce the growing trend of unnecessary surgical procedures was made by Manning, who developed a scientific way to formally diagnose IBS without the need to exclude any other possible disorder first. These criteria were not developed by a single individual, but rather through expert consensus, consensus which later led to the Rome criteria, which has been improved several times until today when it reached its fourth edition (3). The main distinction between The Rome and Manning criteria is that although Rome criteria still recommends that IBS should be a positive diagnosis, they are more restrictive when directly compared to the Manning criteria.

Therefore, when evaluating the symptoms of IBS, the diagnostic criteria which are used are very important to be mentioned as they reflect how the diagnostic is determined and these differences between studies matter when they are evaluated over time. Most studies use either the Manning, Rome III, or Rome IV criteria, and the variation between these can be significant when they are reviewed /analyzed afterwards. However, some authors argue that the Rome criteria are only useful for clinical trials and not for clinical practice. The main argument that these authors have raised is that the criteria have been assembled by only secondary and tertiary care specialists. Their knowledge is not called into question; however, undoubtedly these specialists see a different kind of population of patients compared to those who are seen in primary care. It is also important to be mentioned that the criteria are recommended to be implemented in primary care. All the same, the available literature shows that in general, specialists use other clinical symptoms (stress and bloating are the most common) to diagnose their patients with IBS and do not necessarily use the Rome or Manning criteria (4). That is the main reason why various authors even advocate that diagnosing IBS with the before mentioned sets of criteria should be abandoned and IBS should remain a diagnosis made by excluding other possible disorders (5). However, the International Practice guidelines completely disagree with this idea and state that positive diagnosing should be used with the help of the Rome criteria (6).

Given the importance of Rome criteria, their validity has been broadly studied in the literature. However, on account of the fact that no gold standard (such as a biomarker) exists to allow independent confirmation of IBS, conventional measurement of sensitivity and specificity in general population samples could not be used. Instead of the traditional methods, scientists used estimations of specificity among individuals with organic gastrointestinal disease, and sensitivity amongst patients who have already been diagnosed. The reported results of all these studies show that these criteria have a low specificity to diagnose IBS in individuals with organic gastrointestinal disease. The value of the specificity varies form study to study but the mean is approximately 0.7 (7). The main explanation of this relative low value is that the symptoms experienced in IBS are also 


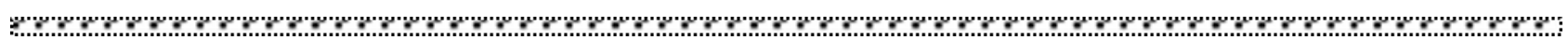

encountered in numerous other

gastrointestinal disorders. It is also important to mention that some authors succeeded in increasing the specificity to 0.9 by extensively investigate patients with specific symptoms such as anaemia, weight loss, and rectal bleeding and by diagnosing IBS by exclusion (8). As before mentioned sensitivity across the criteria varies from study to study; the reported results from the literature show that it ranges from 0.4 to 0.9 . The main factor that leads to this variation in specificity is the experience of the clinician who are using the criteria for positive diagnosing IBS (7).

Therefore, despite the important effort being made, IBS remains hard to diagnose with positive diagnosing or even by excluding any other possible gastrointestinal disorder. As a result, individuals with IBS are often misdiagnosed and have been reported to undergo disproportionately high rates of abdominal and pelvic surgery (9). These high reported rates of misdiagnose and unnecessary surgical procedures require further investigation on the common symptoms of IBS with other disorders that require pelvic and abdominal surgery. We chose to review the available literature in an attempt to understand why IBS is misdiagnosed and uncover the connection of this gastrointestinal disorder with various abdominal and pelvic surgical procedures. To be more specific we will refer to the correlation of IBS with appendectomy, cholecystectomy and hysterectomy.

\section{IBS AND APPENDECTOMY}

The first of the surgery that may be connected with IBS diagnosing is the appendectomy. We chose this procedure for the reason that patients suffering from IBS receive disproportionately high rates of appendectomy (9). This high percentage of patients with IBS who undergo appendectomy is not only obtained in comparison with healthy controls, but also in comparison with patients suffering from various other gastrointestinal disorders. For example, the reported results of a study showed that patients with IBS had four times more chances to undergo appendectomy (34\% compared to $8 \%$ ) when compared to patients with ulcerative colitis (10). Regarding the comparison with the general population, the literature shows that $25-46 \%$ of patients with IBS undergo appendectomy, rates that are much higher when compared to healthy controls (11). Furthermore, another report showed that the prevalence of appendectomy in patients with IBS was a $100 \%$ higher when compared to patients without IBS (12).

However, despite the before mentioned increase in appendectomy rates reported in patients with IBS, there is little data regarding whether the patients in these studies presented pathological evidence of appendicitis postsurgery. To be more specific, although clinical symptoms of appendicitis are often distinct there is a high rate of misdiagnosis reported in the literature. This notion is referred to by specialists as negative appendectomy (NA). There are many studies that reported results which revealed that over $15 \%$ of the performed appendectomies showed no pathological indication of appendicitis post operation $(13,14)$. It is interesting to mention that these rates of NA remained the same over time, despite the development of modern imaging technologies, such as CT or ultrasonography (15).

However, even if IBS might prove to be a significant factor in NA, only one report has studied and showed that IBS might in fact contribute to NA (16). This unique study was conducted on 130 patients with IBS. It is vital to mention however that this study was carried out before the criteria for IBS was created and before modern imaging (CT/ ultrasonography) was used in common medical practice. The reported results of this study showed that $33 \%$ of the IBS suffering patients underwent an appendectomy and most of the removed appendices showed no pathological evidence of appendicitis. It would have been interesting to discover if the prevalence of Rome-IV-defined IBS is higher in patients with NA compared to patients with post-surgery evidence of appendicitis.

Thus, the exact mechanisms through which IBS may predispose to NA are still to be 
\%

elucidated. However, it has been speculate that patients suffering from IBS often present hypersensitivity for visceral sensation. Therefore, this hypersensitivity may play an important role in the potential mechanisms through which IBS is leading to NA.

On the other hand, disturbed visceral sensation is one of the most common factors that lead to IBS symptoms. Furthermore, it has been suggested that IBS results from the induced pain of normal physiological gut stimuli that that is not perceived by normal, healthy individuals (17). The best example for this is the postprandial pain experienced on the entry of a food bolus into the caecum that has been observed in $74 \%$ of the IBS patients (18). This is not a singular example; patients suffering from IBS have also described a wide range of normal visceral stimuli in negatively affective matter (19). Another example is represented by the negative symptoms that are experienced by patients with IBS with rectal balloon distension (20). Therefore, a possible explanation on how IBS may lead to NA is that these patients feel a greater intensity of their pain and tenderness (21). Thus, patients with IBS present a visceral hypersensitivity and amplify their abdominal pain and thus confuse the doctors, and in this way lead to the incorrect diagnosis of appendicitis.

In addition, another possible explanation on the observed higher rate of normal appendices removed from patients with IBS may be related to the fact that patients suffering from this disorder also present a higher frequency of healthcare-seeking, Therefore, not only patients with IBS report greater intensity of the abdominal pain, but also more frequently seek medical attention for it. These factors would finally lead to the removal of normal appendices. Therefore, it is important that the history of IBS in patients suspected of acute appendicitis is taken into consideration before performing the appendectomy procedure. CT scans should also be performed in patients suspected of having appendicitis, especially if these patients also suffer from IBS.

\section{CHOLECYSTECTOMY AND IBS}

The second surgery that patients with IBS undergo in higher percentage compared to normal population is the cholecystectomy. The available studies and clinical reports confirm this hypothesis and the vast majority of them show that IBS patients undergo an excess of cholecystectomy compared to the general population $(9,22)$.

The first explanation on why such a high cholecystectomy rate exists in IBS patients is related to the inappropriate surgical indicators for abdominal symptoms, which are not related to gallstones (23). However, this is not the only available explanation in the literature. Some authors, who failed to find any excess of cholecystectomy in patients who suffered from IBS, hypothesized that it is plausible that the studies which found such high rate have wrongly concluded their data. These authors suggest that the high association between cholecystectomy and IBS may be explained by selection bias of two highly prevalent conditions, IBS and gallstones, which could to be associated by simple hazard especially in the limited samples of IBS suffering patients (24).

However, there are many available studies that report a high rate of cholecystectomy in IBS patients that is higher compared to the rate of cholecystectomy in the general population $(21,22)$. Yet, there are also reported results that are in contrast with these findings. For example, a multinational survey, with a sample of 40.000 subjects, from nine different countries, reported a cholecystectomy rate in IBS patients equal with the rate of the normal population (25).

In addition, another study showed that the incidence of gallstones was not different in IBS and control groups, results that may indicate that patients who suffer from IBS have the same risk of gallstones as the general population (26). However, it cannot be excluded that different factors, such as a larger sample or an early diagnose might have found a higher rate of gallstones in the IBS group compared to the control one. 
Therefore, there are still contradictions on what may be the reason on why IBS suffering patients present a higher rate of cholecystectomy surgeries. A possible explanation on why these increased rates of gallstone disease in subjects with IBS are observed may be that these patients are more frequently submitted to clinical investigations and found to have gallstones more frequently than normal/control individuals who have rarer visits to their local hospitals.

Furthermore, another limitation of many of the before mentioned study may be related to their classification of subjects which was based on symptoms. On perfect example is the recalling of having, or having had, gallstones, recollection that might be wrong and in this way induce recall bias. The same recalling bias is even more likely in the moment when the patients are asked to remember their gastrointestinal symptoms from a period of several years before cholecystectomy. Furthermore, when separate analyses were performed in subjects who were unaware of having gallstones, the reported results showed that no statistically significant difference was found regarding the prevalence of gallstones in the group of patients with IBS and the control group.

In addition, another explanation on the observed high rate of cholecystectomy in IBS population is related to the gastrointestinal disturbances and abdominal pain that is fairly common after the surgical procedure of cholecystectomy. These normal, post-surgery symptoms might have been wrongly attributed by the participants to IBS.

Furthermore, the observed high rate of cholecystectomy in IBS subjects may be explained by the wrong prescription of surgical intervention. There is significant evidence in this regard; available studies indicate that various abdominal surgeries are wrongly prescribed for IBS patients (27). In addition, the observed high cholecystectomy rate could be the effect of incorrectly prescribed surgical intervention for considering IBS symptoms due to possible gallstones.
To conclude this section of our review, although correlation does not necessarily mean causal relationship, having abdominal pain or discomfort may prove to be an important risk factor to wrongly undergo cholecystectomy in patients who suffer from IBS.

\section{IBS AND HYSTERECTOMY}

The last surgery from our review which is associated with IBS is the hysterectomy. Almost every surgical procedure will cause postoperative pain. This pain is usually followed by a reduced capacity for experiencing pain and also by a magnified response to pain. The available literature shows that certain factors can predispose to prolonged and magnified pain after the surgery. The main factors are related to the site and the duration of surgery and the emotional state of the patient (28).

Therefore, abdominal surgeries usually cause a specific, unique type of pain regarding the physiologic and clinical features (28). As we showed in the previous sections and it is also demonstrated in the literature, patients with IBS have a higher surgical rate compared to the general population (12). To be more specific for the present section of our work, the literature shows that a high rate of women with IBS have had a hysterectomy.

The first possible explanation on these observed high rates of this procedure is the simple misdiagnosis of IBS resulting in hysterectomy. The second explanation is the other way around, IBS symptoms developing as a result of hysterectomy. The more complex explanation refers to a possible single disorder which may contribute to symptoms in both gastrointestinal and genitourinary tracts (29).

Regarding the specific common symptoms of these two distinct disorders, studies have showed that constipation and a specific subtype of IBS- pain were more common in individuals who underwent hysterectomy (12). Therefore, the majority of the available studies had succeeded in proving a correlation between these symptoms. For example, a study among women undergoing 


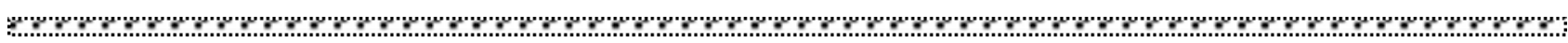

hysterectomy showed that the prevalence of

IBS was significantly higher in the experimental group when compared to the control group (30).

Therefore, the available data shows that many preoperative symptoms which are common in IBS are prevalent in women who are about to undergo hysterectomy. It is important to mention that these symptoms, improve in $60 \%$ of the women after the hysterectomy. This strong correlation with the surgical procedure makes it very unlikely that this association might just be a natural fluctuation of the disorder. Therefore, a more plausible explanation may be related with the preoperative symptoms that may be connected to the effect of the treatment of the gynaecological disorder. Furthermore, the observed improvement of the IBS nonrelated abdominal pain after the hysterectomy may show that this symptom was connected with the gynaecological disorder. In addition, another explanation of the observed improvement of bowel function is that this improvement may result from the withdrawal of treatment that was prescribed for the gynaecological disorder. For example, dysfunctional bleeding and fibroids were the most common symptoms that led to the prescription of hysterectomy. The standard medication treatment for these disorders includes iron supplements, mefenamic acid, oestrogens and progestogens. All of these medications are known to produce bowel dysfunctions as a side effect (31).

The next explanation on the observed improvement in symptoms after hysterectomy is related to the placebo- effect. The placebo effect was demonstrated to be statistically significant in IBS clinical trials (32). However there is a limitation in regards to the placebo effect. Although the placebo effect might explain the observed decrease in abdominal pain after surgery, it seems very unlikely that the women from these studies would believe that IBS-like symptoms would decrease after the hysterectomy.

In addition, other studies have found that gastrointestinal symptoms after the hysterectomy, which occurred with a frequency of more than once per week, were seen in $10 \%$ of the IBS patients (32). Although many studies have been made on the subject the exact reason why hysterectomy can cause IBS symptoms in some women is still unclear.

Another possible explanation is again related to the medicated intervention before surgery, this time. Therefore the use of antibiotics preoperation may determine IBS symptoms by causing a change in bowel flora (33). However, this explanation is not supported by the fact that no less than $60 \%$ of the women in some studies, who had no IBS-like symptoms pre-surgery, received an antibiotic treatment. The results of these studies reported that no association was observed between antibiotic treatment and the development of any symptoms related to bowel dysfunction.

The next possible explanation is related to the psychological factors that may play an important role in the development of new symptoms. The available data suggests that hysterectomy is associated with the onset of anxiety and depression (34). Furthermore, it is also demonstrated that certain psychological disorders are common in IBS individuals (35). However, this causation has yet to be demonstrated. Available studies only succeeded to demonstrate that psychopathology may influence whether patients have the symptoms rather than this disorder can directly cause negative psychological symptoms (36).

Regarding the specificity of the IBS symptoms, constipation is the most frequent bowel disturbance reported after hysterectomy. The exact mechanisms by which hysterectomy may cause constipation are yet to be discovered. Some authors have speculated that the observed dysfunction found in the rectosigmoid motility, accompanied by bladder and bowel dysfunction in patients with constipation after hysterectomy, have led to the hypothesis that autonomic denervation of the pelvic viscera may be a side effect of the surgical procedure (37). However, even if radical hysterectomy 


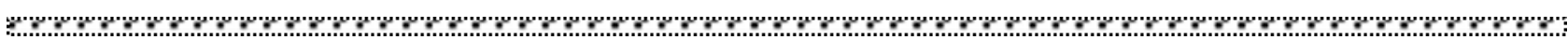

may cause damage to pelvic nerves, these damages do not occur after abdominal or vaginal hysterectomy, surgical procedures that also are demonstrated to induce constipation in some women.

In addition, another potential mechanism through which hysterectomy could be implicated in the development of the IBS symptom may be related to the hormonal changes that are reported after the surgery. This observed decrease in plasma oestrodiol and its precursors, which is common after hysterectomy, has also been reported in women with constipation (38). However, the standard treatment after hysterectomy includes hormone replacement therapy and therefore, this makes very unlikely the assumption that these women may be oestrogen deficient.

It is also to be determined if the decreased level of prostaglandin that are common after hysterectomy could also cause IBS -like symptoms in these women (39). The removal of the uterus, which is an important source of prostaglandins, may cause constipation given the fact that prostaglandins tend to also increase bowel frequency.

In the conclusion of this section, the available data that we analyzed shows that certain IBSlike symptoms that are present before surgery improve after hysterectomy. However, after the procedure $10 \%$ of the operated women, who were asymptomatic before surgery, present gastrointestinal distress, most often in the form of constipation.

\section{THE POSSIBLE RELEVANCE OF IBS- ASSOCIATED PSYCHIATRIC AND PSYCHOLOGICAL SYMPTOMS IN THE PRESENT CONTEXT}

We could also mention in the present context that an increased relevance could be exerted by the well-known and previously demonstrated IBS-associated psychiatric and psychological symptoms, such as anxiety, depression-like manifestations or sleep deficiencies $(43,44)$.

This could have an increased importance since the connection between the general surgery and the psychiatric/psychological complications associated with them are understudied in present research literature (45), with these aspects being suggested in most types of surgery, including the bariatric one (46).

Also, in addition it could speculated that oxidative stress could have an increased relevance in this context, since it can be further connected with both IBS (47-49), most of the neuropsychiatric disorders (50) and also the surgical process in general (51).

\section{CONCLUSIONS}

Although the prevalence of IBS in the general population is approximately $10-15 \%$, more than half of patients that seek evaluation from the doctors regarding their pelvic pain also suffer from IBS. Furthermore, more than $33 \%$ of patients who are prescribed a surgical procedure, and also present a non-specific abdominal pain are also diagnosed with IBS (40). In addition, individuals who suffer from IBS also report a high prevalence of various abdominal and pelvic surgeries (41).

Therefore, an important problem arises when many IBS patients who are complaining of various gastrointestinal symptoms, undergo abdominal and pelvic surgery and post-surgery display normal histological findings of the surgically resected tissue (42). These observations demonstrate that some of the IBS symptoms are misinterpreted as indicators of surgically remediable disorders. Further research is vital in order to educate physicians and prevent unnecessary surgeries in IBS patients. From these observations we can understand the importance of systematically reviewing the research on the association between IBS and abdominal and pelvic surgeries for a better comprehension of how this chronic gastrointestinal disorder interacts with other medical conditions. 


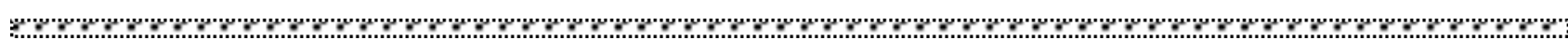
Also, there could be an increased relevance for the IBS-associated psychiatric and psychological symptoms in this context, which could result in even further complications in this context.

\section{ACKNOWLEDGEMENTS AND DISCLOSURES}

AC is supported by a research grant for Young Teams offered by UEFISCDI Romania, no. PNIIIP1-1.1-TE-2016-1210, contract no. 58 from 02/05/2018, called "Complex study regarding the interactions between oxidative stress, inflammation and neurological manifestations in the pathophysiology of irritable bowel syndrome (animal models and human patients)"

\section{REFERENCES}

1. Chaudhary NA, Truelove SC. The irritable colon syndrome. A study of the clinical features, predisposing causes, and prognosis in 130 cases. Q J Med. 1962;31:307-322

2. Kruse FH. Functional disorders of the colon: the spastic colon, the irritable colon, and mucous colitis. Cal West Med. 1933;39(2):97-103.

3. Longstreth GF, Thompson WG, Chey WD, Houghton LA, Mearin F, Spiller RC. Functional bowel disorders. Gastroenterology. 2006;130(5):1480-1491.

4. Mearin F, Lacy BE. Diagnostic criteria in IBS: useful or not? Neurogastroenterol Motil. 2012;24(9):791-801. .

5. Quigley EM. The 'con' case. The Rome process and functional gastrointestinal disorders: the barbarians are at the gate! Neurogastroenterol Motil. 2007;19(10):793-797.

6. Quigley EMM, Fried M, Gwee K-A, et al. Irritable Bowel Syndrome: A Global Perspective. Milwaukee (WI): World Gastroenterology Organisation; 2009:1-20.

7. Whitehead WE, Palsson OS, Feld AD, et al. Utility of red flag symptom exclusions in the diagnosis of irritable bowel syndrome. Aliment Pharmacol Ther. 2006;24(1):137-146.

8. Whitehead WE, Drossman DA. Validation of symptom-based diagnostic criteria for irritable bowel syndrome: a critical review. Am J Gastroenterol. 2010;105(4):814-820;

9. Hasler WL, Schoenfeld P. Systematic review; abdominal and pelvic surgery in patients with irritable bowel syndrome. Aliment Pharmacol Ther. 2003;17:997-1005

10. Burns DG. The risk of abdominal surgery in irritable bowel syndrome. S Afr Med J. 1986;70:91.

11. Fielding JF, Bianchi P, Brown JM, et al. The O.M.G.E. Irritable Bowel Syndrome Survey. Scand J Gastroenterol Suppl. 1984;95:70-2.

12. Longstreth GF, Yao JF. Irritable bowel syndrome and surgery: a multivariable analysis. Gastroenterology. 2004;126:1665-73.

13. Korner H, Sondenaa K, Soreide JA, et al. Incidence of acute nonperforated and perforated appendicitis: age-specific and sex-specific analysis. World J Surg. 1997;21:313-17.

14. Flum DR, Morris A, Koepsell T, et al. Has misdiagnosis of appendicitis decreased over time? A population-based analysis. JAMA. 2001;286:1748-53.

15. Flum DR, McClure TD, Morris A, et al. Misdiagnosis of appendicitis and the use of diagnostic imaging. J Am Coll Surg. 2005;201:933-9.

16. Liu CC, Lu CL, Yen DH, et al. Diagnosis of appendicitis in the ED: comparison of surgical and nonsurgical residents. Am J Emerg Med. 2001;19:109-12.

17. Hasler WL, Owyang C. Irritable bowel syndrome, 3rd edn. Philadelphia: Lippincott Williams \& Wilkins, 1999.

18. Cann PA, Read NW, Brown C, et al. Irritable bowel syndrome: relationship of disorders in the transit of a single solid meal to symptom patterns. Gut. 1983;24:405-11.

19. Naliboff BD, Munakata J, Fullerton S, et al. Evidence for two distinct perceptual alterations in irritable bowel syndrome. Gut. 1997;41:505-12.

20. Whitehead WE, Engel BT, Schuster MM. Irritable bowel syndrome: physiological and psychological differences between diarrhea-predominant and constipationpredominant patients. Dig Dis Sci. 1980;25:404-13.

21. Andersson RE, Hugander AP, Ghazi SH, et al. Why does the clinical diagnosis fail in suspected appendicitis? Eur J Surg. 2000;166:796-802.

22. Havia T, Manner R. A follow-up study with special reference to the development of diverticula. Acta Chir Scand. 1971;137:569-72.

23. Smith RC, Greenbaum DS, Vancouver JB, Henry RC, Reinhart MA, Greenbaum RB, et al. Psychosocial factors are associated with health care seeking rather than diagnosis of irritable bowel syndrome. Gastroenterology. 1990;98:293301 .

24. Hungin APS, Whorwell PJ, Tack J, Mearin F. The prevalence, patterns and impact of irritable bowel syndrome: an international survey of 40000 subjects. Aliment Pharmacol Ther. 2003;17:643-50.

25. Longstreth SF, Wolde-Tsadik G. Irritable bowel-type symptoms in HMO examinees. Prevalence, demographics, and clinical correlates. Dig Dis Sci. 1993;38:1581-9.

26. Kennedy TM, Jones RH. Epidemiology of cholecystectomy and irritable bowel syndrome in a UK population. Br J Surg. 2000;87:1658-63. 


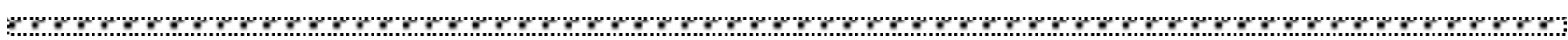
27. Gambone JC, Reiter RC. Nonsurgical management of chronic pelvic pain. A multidisciplinary approach. Clin Obstet Gynecol. 1990;33:205-11.

28. Bonica JJ. Current status of postoperative pain therapy. In: Yokota T, Dubner R, eds. Current topics in pain research and therapy. Amsterdam, The Netherlands: Excerpta Medica. 1983;169.

29. Kennedy TM, Jones RH. The epidemiology of hysterectomy and irritable bowel syndrome in a UK population. Int J Clin Pract. 2000;54:647-50.

30. Sperber AD, Morris CB, Greemberg L, Bangdiwala SI, Goldstein D, Sheiner E, et al. Development of abdominal pain and IBS following gynecological surgery: a prospective, controlled study. Gastroenterology. 2008;134:75-84.

31. British National Formnularv. British Medical Association and The Pharmaceutical Society of Great Britain. 1990.

32. Klein KB. Controlled treatment trials in the irritable bowel syndrome: a critique. Gastroenterology. 1988;95:232-41.

33. Alun Jones V, Hunter JO. Irritable bowel syndrome and Crohn's disease. In: Brostoff J, Challacombe T, eds. Food allergy and intolerance. London: Bailliere Tindall. 1987:555-69.

34. Oates M, Gath D. Psychological aspects of gynaecological surgery. Baillieres Clin Obstet Gvnaecol. 1989;3:729-49.

35. Creed F, Guthrie E. Psychological factors in the irritable bowel syndrome. Gut. 1987;28:1307-18.

36. Whitehead WE, Bosmajian L, Zonderman AB, Costa PT, Schuster MM. Symptoms of psychological distress associated with irritable bowel syndrome. Comparison of community and medical clinic samples. Gastroenterologv. 1988;95:709-14.

37. Varma JS, Smith AN. Abnormalities of colo-rectal function in intractable constipation following hysterectomy. Gut. 1985;26:A581-2.

38. Mundy AR. An anatomical explanation for bladder dysfunction following rectal and uterine dvsfunction. Br J Urol. 1982;54:501-4.

39. Kamm MA, Lennard-Jones JE, Farthing MJG, McILean A, Perry L, Chard T. Women with intractable constipation have an abnormalitv of adrenal steroid. Gut. 1989;30:A75-1.

40. Drossman DA, Li Z, Andruzzi E, et al. US householder survey of functional gastrointestinal disorders: prevalence, sociodemography and health impact. Dig Dis Sci. 1993;38:1569-80.

41. Chaudhary NA, Truelove SC. The irritable colon syndrome: a study of the clinical features, predisposing causes, and prognosis in 130 cases. Q J Med. 1962;31:307-23.

42. Longstreth GF, Preskill DB, Youkeles L. Irritable bowel syndrome in women having diagnostic laparoscopy or hysterectomy: relation to gynecologic features and outcome. Dig Dis Sci. 1990;35:1285-90.

43. Balmus IM, Robea M, Ciobica A, Timofte D. Perceived stress and gastrointestinal habits in college students. Acta Endocrinol (Buchar). 2019;15(2):274-275.

44. Balmus IM, Cojocariu RO, Ciobica A, et al. Preliminary Study on the Tears Oxidative Stress Status and Sleep Disturbances in Irritable Bowel Syndrome Patients. Oxid Med Cell Longev. 2020;2020:4690713.

45. Abrams TE, Vaughan-Sarrazin M, Rosenthal GE. Influence of Psychiatric Comorbidity on Surgical Mortality. Arch Surg. 2010;145(10):947-953.

46. Kubik JF, Gill RS, Laffin M, Karmali S. The impact of bariatric surgery on psychological health. J Obes. 2013;2013:837989.

47. Balmus IM, Lefter R, Ciobica A, et al. Preliminary Biochemical Description of Brain Oxidative Stress Status in Irritable Bowel Syndrome Contention-Stress Rat Model. Medicina (Kaunas). 2019;55(12):776.

48. Balmus IM, Ilie-Dumitru O, Ciobica A, et al. Irritable Bowel Syndrome between Molecular Approach and Clinical Expertise-Searching for Gap Fillers in the Oxidative Stress Way of Thinking. Medicina (Kaunas). 2020;56(1):38.

49. Balmus IM, Ciobica A, Cojocariu R, Luca AC, Gorgan L. Irritable Bowel Syndrome and Neurological Deficiencies: Is There A Relationship? The Possible Relevance of the Oxidative Stress Status. Medicina (Kaunas). 2020;56(4):175

50. Balmus IM, Ciobica A, Antioch I, Dobrin R, Timofte D. Oxidative Stress Implications in the Affective Disorders: Main Biomarkers, Animal Models Relevance, Genetic Perspectives, and Antioxidant Approaches. Oxid Med Cell Longev. 2016;2016:3975101.

51. Kücükakin B, Gögenur I, Reiter RJ, Rosenberg J. Oxidative stress in relation to surgery: is there a role for the antioxidant melatonin?. J Surg Res. 2009;152(2):338-347.

52. Rosenfeldt F, Wilson M, Lee G, et al. Oxidative stress in surgery in an ageing population: pathophysiology and therapy. Exp Gerontol. 2013;48(1):45-54.

\section{Correspondence:}

Peiu Sorin Nicolae,

MD, Faculty of Medicine, "Grigore T. Popa" University of Medicine and Pharmacy Iasi, Romania, sorinpeiu@gmail.com 